\title{
Asociación entre factores ambientales \\ y pautas de crianza con el Índice de Masa Corporal de preescolares de hogares infantiles de Floridablanca, Colombia
}

\author{
Relationship between environmental factors \\ and child rearing practices on body mass index \\ of preschool children residents of children's \\ homes from Floridablanca, Colombia
}

\section{ABSTRACT}

The association of environmental factors and child rearing practices with body mass index z-score (BMIZ) in children residents of Children's Homes from Floridablanca, Colombia was studied. A random sample of 176 preschool children aged 3-6 years was weighed and measured, their parents or caregiver were also measured and they answered a survey. The generalized linear model showed that BMIZ increased significantly with children's age $(\beta=0.02)$, children's sex (male $\beta=0.27$ ), mother's occupation (homemaker $\beta=0.29$ ), mother's overweight $(\beta=0.20)$, person who accompanies children while they eat (relatives $\beta=0,49$; anyone or non-relative $\beta=0.40$ ), person who cooks (grandmother $\beta=0.55)$, adequate time spent on eating by children $(\beta=0.54)$, and overeating (0.51). BMIZ decreased significantly with mother's occupation (looking for a job or student $\beta=-0.43$ ), maternal history of hypertension $(\beta=-0.26)$, motivation to eat $(\beta=-0.59)$ and pressure to eat $(\beta=-0.55)$.

Key words: Child rearing, risk factors, body mass index, pediatric obesity, feeding behavior.
Gloria E Prada (1,2)

Mónica P. Gutierrez (3)

Ximena L. Sánchez (2)

Laura P. Rueda (2)

Adriana Angarita (2)

(1) Observatorio Epidemiológico de Enfermedades Cardiovasculares. Centro de Investigaciones Epidemiológicas. Universidad Industrial de Santander, Bucaramanga, Colombia. (2) Escuela de Nutrición y Dietética.

Universidad Industrial de Santander, Bucaramanga, Colombia. (3) Centro Zonal Bucaramanga Sur, Instituto Colombiano de Bienestar Familiar (ICBF), Regional Santander, Colombia.

Dirigir la correspondencia: Profesora

Gloria Esperanza Prada Gómez Centro de Investigaciones Epidemiológicas Facultad de Salud, Universidad Industrial de Santander Carrera 32 No. 29-31, tercer piso Bucaramanga, Colombia Teléfax: (57-7) 6323215 E-mail:pradauis@gmail.com gprada@uis.edu.co

Este trabajo fue recibido el 16 de Mayo de 2014 y aceptado para ser publicado el 15 de Julio de 2014.

y $20,2 \%$ sobrepeso (> $1 \mathrm{a} \leq 2 \mathrm{DE}$ ) (4).

La obesidad infantil se asocia a dos de los 3 periodos del

La prevalencia de obesidad en la infancia aumentó entre 1,1 y 4,6 veces desde la década de los 70 hasta la década de los 90 en países como Australia, Brasil, Canadá, Chile, Finlandia, Francia, Alemania, Grecia, Japón, Inglaterra y Estados Unidos (1). El año 2010, la prevalencia de sobrepeso y obesidad fue más de $40 \%$ en Norte América y en regiones del oriente del mediterráneo, 38\% en Europa, 27\% en la región occidental del Pacífico y $22 \%$ en el sudeste de Asia (2). En Latinoamérica, Rivera y col. estimaron una prevalencia de sobrepeso de $7,1 \%$ en niños menores de cinco años, según la clasificación de la Organización Mundial de la Salud con el indicador peso para la talla. Así mismo, estimaron que 3,8 millones de niños menores de 5 años tienen sobrepeso u obesidad (3). En Colombia, $5,2 \%$ de los menores de cinco años tiene obesidad (> $2 \mathrm{DE}$ ) incremento del tejido adiposo; el primero se presenta de los 8 a 9 meses de vida, el segundo alrededor de los 5 o 6 años de edad y el tercero en la pre-adolescencia y en el estirón puberal. El mayor riesgo de presentar obesidad está en el segundo periodo (5), por eso la etapa preescolar es crítica para el inicio de la obesidad infantil y es la más indicada para iniciar programas de prevención y fortalecer conductas saludables (6).

Al respecto, Moraga et al., en un estudio prospectivo de 8 meses de seguimiento, encontraron que los niños que iniciaron control de peso antes de los 5 años de edad obtuvieron mayor porcentaje de baja de peso que quienes iniciaron su control después de los 5 años (7). Por otro lado, la evidencia muestra como en la etapa preescolar los padres y cuidadores afectan 
positiva o negativamente el comportamiento, la personalidad y la salud física de los niños $(8,9)$.

El aumento de la obesidad infantil requiere con urgencia estudios que permitan conocer los factores asociados, especialmente los de tipo ambiental y ecológico que son poco estudiados y que se constituyen en un insumo fundamental para la formulación de políticas. Según estudios internacionales, los elementos determinantes que constituyen el entorno social del niño obeso son, los hábitos familiares, el ambiente del colegio, la disponibilidad de alimentos altos en energía, la falta de tiempo para comer en familia, influencia de la publicidad y estilos de crianza (8-12). Estos últimos, están mediados por factores culturales e impactan el comportamiento alimentario y el estado nutricional de los niños $(8,9)$.

A pesar de lo expuesto anteriormente, pocos estudios han establecido cuáles factores están asociados al exceso de peso en preescolares residentes en Colombia. Es por esto que el objetivo de este trabajo fue establecer los factores ambientales y estilos de crianza asociados al Indice de Masa Corporal (IMC) en niños y niñas usuarios del programa de Hogares Infantiles (HI) del Instituto Colombiano de Bienestar Familiar (ICBF) localizados en el municipio de Floridablanca, Santander, Colombia.

\section{SUJETOS Y MÉTODOS}

Estudio de corte transversal realizado durante el año 2012 y 2013 . El universo estuvo constituido por 665 niños y niñas de tres a seis años, usuarios del programa de HI del ICBF. Se incluyeron preescolares inscritos y con asistencia regular a los siete $\mathrm{HI}$ existentes en la zona urbana de Floridablanca, municipio del área metropolitana de Bucaramanga con una población estimada para el 2012 de 271,728 habitantes; adicionalmente, los preescolares debían contar con un informante (representante legal o persona a cargo del menor). Se calculó un tamaño muestral de 174 preescolares, con el $10 \%$ de pérdida, se consideró un nivel de confianza del $95 \%$, poder de $80 \%$ y una precisión del $5,0 \%$ y una prevalencia de exceso de peso esperada de 16,0\% (13). Para este análisis se excluyeron 31 participantes con riesgo de talla baja o talla baja medido a través del indicador talla para la edad y sexo ( $z>1 \mathrm{a} \geq 2 \mathrm{DE}$ y $>2 \mathrm{DE}$ ), para un total de 176 preescolares.

La selección de la muestra se realizó mediante muestreo aleatorio estratificando por los siete HI. Antes de recolectar la información, se realizó la prueba piloto en un grupo de 20 niños y niñas con características similares a la población de estudio.

Seis estudiantes de último año del programa de Nutrición y Dietética de la Universidad Industrial de Santander, entrenados en métodos de entrevista directa y mediciones antropométricas aplicaron una encuesta, elaborada por el equipo de investigación (no validada), que indagaba por factores biológicos, económicos, sociodemográficos del niño(a) y sus familiares, antecedentes patológicos familiares de los padres, parentesco de la persona encargada del cuidado del niño(a) y estilos de crianza relacionados con alimentación. Se destaca que la estratificación socioeconómica colombiana, es una clasificación de los domicilios o viviendas a partir de sus características físicas y de su entorno, en seis grupos o estratos: 1. Bajo-bajo, 2. Bajo, 3. Medio-bajo, 4. Medio, 5. Medio-alto, 6. Alto (14).

Así mismo realizaron las mediciones de peso y talla a los preescolares, madre y padre, siguiendo los protocolos de la ISAK (Internacional Standards for Anthropometrics Assessment) y se utilizaron básculas digitales marca Tanita HD-
314 con capacidad Máxima 150 kg, con calibración automática y una precisión de $0.1 \mathrm{~kg}$, tallímetros marca SECA Modelo 206. Las encuestas y mediciones se realizaron durante los meses de septiembre a noviembre del año 2012.

La variable dependiente fue el puntaje z del IMC para la edad y sexo, indicador que se construyó utilizando el programa ANTHRO (15). Una vez obtenida la muestra, los datos fueron ingresados al software EPIDATA 3.1 (16), la base de datos resultante fue exportada al software estadístico Stata (17) para su respectivo análisis. Las variables cualitativas se presentaron en frecuencias absolutas y relativas; en las variables cuantitativas se calculó la media aritmética y la desviación estándar, previa comprobación de normalidad con la prueba de Shapiro-Wilk. Si lo anterior no fuese comprobado, se usó la mediana y el rango intercuartílico (RIC). En el análisis bivariado se utilizó la prueba rango con sumas de Wilcoxon para datos independientes cuando no hubo distribución normal y prueba t de student cuando los datos se ajustaron a una distribución normal (18). Se aplicaron modelos lineales generalizados (MLG) múltiples con el fin de establecer si existían variables asociadas al puntaje $z$ del IMC para la edad y sexo. En el modelo se usó una dispersión de dos veces el promedio y el método cuasi-likelihood. El MLG es un modelo semiparamétrico para datos heterogéneos que no cumplen los supuestos de normalidad, adicionalmente por el método cuasi-likelihood no es necesario especificar la distribución de la variable respuesta; la interpretación de los coeficientes es directa y se logra el ajuste de sobredispersión de los datos (19). La evaluación del modelo final se realizó mediante la prueba linktest y se realizó el análisis de residuales deviance.

Según la Resolución No. 008430 del 04 de octubre de 1993 del Ministerio de Salud de Colombia (20), esta investigación se consideró sin riesgo. Se garantizaron los principios éticos de respeto, autonomía, justicia y confidencialidad. El presente estudio fue aprobado por el Comité de ética para la investigación científica de la Facultad de Salud de la Universidad Industrial de Santander y se solicitó el consentimiento informado por escrito del representante legal de los menores.

\section{RESULTADOS}

La muestra quedó conformada por 80 niñas y 96 niños, el promedio de edad fue de 52,0 \pm 9,7 meses, predominó el grado escolar prejardín con $47,7 \%$ y los estratos socioeconómicos 1 a 2 con $62,5 \%$ (tabla1).

En las características familiares destaca que $62,5 \%$ de los hogares estaba conformado hasta por cuatro personas y $84,1 \%$ de los hogares había sólo un menor de 7 años. El 55,1\% de las familias recibía más de 3 salarios mínimos legales vigentes para 2012 (SMLV), una tercera parte de las madres vivía en unión libre $(35,2 \%)$ o casada $(33,5 \%)$ y $75,6 \%$ de ellas trabajaba. En $27,3 \%$ de los hogares había historial de divorcio, 38,6\% de los preescolares vivía con un solo padre. En 49,4\% de los casos los dos padres aportaban al sostenimiento del hogar (tabla 1). La mediana del puntaje Z del IMC para la edad y sexo en la muestra fue de 0,56 con un mínimo de $-2,13$ y un máximo de 5,47; para las niñas fue de 0,46 con un mínimo de $-1,76$ y un máximo de 2,98 y para los niños fue de 0,65 con un mínimo de $-2,13$ y un máximo de 5,47.

En el análisis bivariado del IMC para edad y sexo, se encontró un aumento significativo del puntaje $z$ con la edad $(\beta=0,03)$, sexo masculino $(\beta=0,38)$, escolaridad superior (Jardín $\beta=0,40$ y transición $\beta=0,94$ ) y ocupación de la madre: hijos de madres dedicadas al oficio del hogar comparado con las mujeres que estaban trabajando $(\beta=0,43)$. Por el contrario, 
TABLA 1

Características sociodemográficas y familiares de una muestra de niños y niñas de los Hogares Infantiles del ICBF asociadas al puntaje z IMC para edad y sexo. Floridablanca, Colombia, 2012.

\begin{tabular}{|c|c|c|c|c|c|c|}
\hline \multirow{3}{*}{$\begin{array}{c}\text { Variable } \\
\text { Edad (meses) Promedio } \pm \mathrm{DE}\end{array}$} & \multicolumn{2}{|c|}{ Medida de resumen } & \multirow{3}{*}{$\begin{array}{c}\text { IC95\% } \\
50,8-53,7\end{array}$} & \multirow{3}{*}{$\begin{array}{c}\text { Coeficiente Beta } \\
\qquad 0,03\end{array}$} & \multirow{3}{*}{$\begin{array}{c}\mathrm{IC95 \%} \\
0,02 ; 0,04\end{array}$} & \multirow{3}{*}{$\begin{array}{l}\text { Valor } p \\
0,000\end{array}$} \\
\hline & 52,0 & $\pm 9,7$ & & & & \\
\hline & & & & & & \\
\hline Femenino & 80 & 45,5 & $38,0-52,9$ & & & \\
\hline Masculino & 96 & 54,5 & $47,1-62,0$ & 0,38 & 0,$19 ; 0,57$ & 0,000 \\
\hline \multicolumn{7}{|l|}{ Grado escolar n \% } \\
\hline Prejardin & 84 & 47,7 & $40,3-55,2$ & & & \\
\hline Jardín & 75 & 42,6 & $35,2-50,0$ & 0,40 & 0,$20 ; 0,59$ & 0,000 \\
\hline Transición & 17 & 9,7 & $5,3-14,1$ & 0,94 & 0,$61 ; 1,27$ & 0,000 \\
\hline \multicolumn{7}{|l|}{$\begin{array}{l}\text { Número de personas que conforman } \\
\text { el núcleo familiar } n \%\end{array}$} \\
\hline $1-4$ & 110 & 62,5 & $55,3-69,7$ & & & \\
\hline $5-9$ & 66 & 37,5 & $30,3-44,7$ & $-0,01$ & $-0,21 ; 0,17$ & 0,853 \\
\hline \multicolumn{7}{|l|}{$\begin{array}{l}\text { Número de personas que viven con } \\
\text { el niño(a) (sin incluirlo) } n \%\end{array}$} \\
\hline $1-3$ & 101 & 57,4 & $50,0-64,7$ & & & \\
\hline $4-9$ & 75 & 42,6 & $35,2-50,0$ & $-0,19$ & $-0,39 ;-0,005$ & 0,044 \\
\hline \multicolumn{7}{|l|}{ Número de niños y niñas menores de } \\
\hline \multicolumn{7}{|l|}{7 años que viven en la casa $n \%$} \\
\hline 1 & 148 & 84,1 & $78,6-89,5$ & & & \\
\hline $2-4$ & 28 & 15,9 & $10,5-21,4$ & $-0,09$ & $-0,35 ; 0,17$ & 0,485 \\
\hline \multicolumn{7}{|l|}{ Estado civil de la madre $\mathrm{n} \%$} \\
\hline Unión Libre & 62 & 35,2 & $28,1-42,4$ & & & \\
\hline Casada & 59 & 33,5 & $26,5-40,6$ & $-0,07$ & $-0,30 ; 0,16$ & 0,534 \\
\hline Separada/ Divorciada/Viuda n \% & 12 & 6,8 & $3,1-10,6$ & 0,29 & $-0,10 ; 0,69$ & 0,155 \\
\hline Madre Soltera & 43 & 24,4 & $18,0-30,8$ & $-0,13$ & $-0,38 ; 0,12$ & 0,300 \\
\hline \multicolumn{7}{|l|}{ Ocupación de la madre } \\
\hline Trabajando & 133 & 75,6 & $69,2-82,0$ & & & \\
\hline Buscando trabajo/estudiando & 11 & 6,3 & $2,6-9,9$ & $-0,55$ & $-0,93 ;-0,15$ & 0,006 \\
\hline Oficios del hogar & 20 & 11,4 & $6,6-16,1$ & 0,43 & 0,$13 ; 0,72$ & 0,004 \\
\hline \multicolumn{7}{|l|}{ Historial de divorcio } \\
\hline No & 116 & 65,9 & $58,8-72,9$ & & & \\
\hline $\mathrm{Si}$ & 48 & 27,3 & $20,6-33,9$ & 0,11 & $-0,11 ; 0,33$ & 0,310 \\
\hline Sin información & 12 & 6,8 & $3,1-10,6$ & 0,06 & $-0,13 ; 0,25$ & 0,564 \\
\hline \multicolumn{7}{|l|}{ Actualmente el niño (a) vive con } \\
\hline \multicolumn{7}{|l|}{ Ambos n \% padres } \\
\hline No & 68 & 38,6 & $31,4-45,9$ & & & \\
\hline $\mathrm{Si}$ & 108 & 61,4 & $54,1-68,6$ & 0,06 & $-0,14 ; 0,25$ & 0,564 \\
\hline \multicolumn{7}{|l|}{ Cuidador } \\
\hline Madres & 118 & 67,1 & $60,0-74,1$ & & & \\
\hline Padres & 27 & 15,3 & $10,0-20,7$ & 0,44 & 0,$17 ; 0,70$ & 0,001 \\
\hline Abuelos/tios/Otros & 31 & 17,6 & $11,9-23,3$ & 0,32 & 0,$07 ; 0,58$ & 0,013 \\
\hline \multicolumn{7}{|l|}{ Estrato socioeconómico n \% } \\
\hline $1-2$ & 110 & 62,5 & $55,3-69,7$ & & & \\
\hline $3-5$ & 66 & 37,5 & $30,3-44,7$ & $-0,04$ & $-0,23 ; 0,16$ & 0,677 \\
\hline \multicolumn{7}{|l|}{ Ingresos mensuales n \% } \\
\hline $0-2$ SMLV & 78 & 44,3 & $36,9-51,7$ & & & \\
\hline$>3$ SMLV & 97 & 55,1 & $47,7-62,5$ & 0,32 & 0,$13 ; 0,51$ & 0,001 \\
\hline \multicolumn{7}{|l|}{$\begin{array}{l}\text { ¿Quién aporta para el sustento } \\
\text { del hogar? }\end{array}$} \\
\hline Padre & 30 & 17,1 & $11,4-22,7$ & & & \\
\hline Madre & 36 & 20,5 & $14,4-26,5$ & $-0,31$ & $-0,62 ; 0,004$ & 0,053 \\
\hline Padre y madre & 87 & 49,4 & $42,0-56,9$ & $-0,18$ & $-0,44 ; 0,09$ & 0,187 \\
\hline Otros & 23 & 13,1 & $8,0-18,1$ & $-0,01$ & $-0,36 ; 0,34$ & 0,971 \\
\hline
\end{tabular}


se observó una disminución en el puntaje z del IMC para la edad y sexo en las familias donde vivían entre 4 y 9 integrantes sin incluir el niño $(\beta=-0,19)$ comparado con las familias de menos de 4 integrantes; así como en los niños de madres que estaban estudiando $(\beta=-0,82)$ comparado con los hijos de madres trabajadoras (tabla 1).

Respecto a los antecedentes patológicos de la madre (tabla 2), las mayores prevalencias fueron, la hipertensión arterial con $33,0 \%$ y la diabetes para las madres con $29,0 \%$. Se encontró que los antecedentes de obesidad $(\beta=0,33)$ aumentan significativamente el puntaje z del IMC; mientras que, los antecedentes de diabetes $(\beta=-0,49)$ e hipertensión arterial $(\beta=-0,32)$ lo disminuyen.

En relación con los estilos de crianza, 38,6\% de los cuidadores reportó que en las comidas principales le ofrece cantidades adicionales, $94,3 \%$ se dedicaba a comer con el niño(a), 63,6\% motivaban al preescolar cuando deja de comer y consideran que no ha comido lo suficiente. En la pregunta ¿Cuándo el niño no come lo que usted le ofrece, que hace usted?, 11,9\% lo motivaba, ofrecía otro alimento, dialogaba, $22,7 \%$ respondió que lo presiona. Por otra parte, el $41,5 \%$ indicó que promueve el consumo de alimentos mediante palabras y diálogo; mientras que, 32,4\% recurrió a la presión mediante chantaje o regaño (tabla 3 ).

Mientras consume los alimentos, $45,0 \%$ de los preescolares estaban acompañados por un familiar y $42,1 \%$ por cualquiera de sus dos padres. Los responsables de la preparación de alimentos fueron, la madre en $59,7 \%$ de los casos y la abuela en $27,8 \%$, y en una proporción similar son responsables de ofrecer la alimentación de lunes a sábado, el domingo la participación de la madre fue mayor (71,6\%). La decisión del tipo de alimentos que debe consumir el niño(a) era tomada principalmente por la madre (71,0\%), sin embargo $17,6 \%$ de las abuelas, respondían por esta decisión. Según la percepción del cuidador, 35,8\% de los niños rechazaba la comida, comía sin apetito o jugaba con ella; respecto al tiempo empleado para el consumo de alimentos, el cuidador manifestó que un 43,2\% de los preescolares comía despacio y 6,3\% rápido; por otro lado, cuando está sano, $11,4 \%$ de los encuestados refirió que el niño(a) come demasiado y $12,5 \%$ que come poco (tabla 3 ).

Se encontró un aumento significativo del puntaje $z$ del IMC para la edad y sexo en los preescolares que eran acompañados por un familiar $(\beta=0,76)$, que comían solos o con otra persona no familiar $(\beta=0,81)$, comparado con aquellos que estaban acompañados por cualquiera de los padres; en los niños que no se motivan o no es necesario hacerlo para que coman $(\beta=0,50)$, en los niños a quienes la abuela les preparaba el alimento $(\beta=0,25)$; y en aquellos niños en que los alimentos son ofrecidos el sábado $(\beta=0,26)$ y el domingo $(\beta=0,31)$ por la abuela u otra persona (Sábado: $\beta=0,40$ y Domingo: $\beta=0,37$ ), cuando la persona que decide los alimentos que consume el preescolar era otra diferente a la madre $(\beta=0,48)$, en los niños que según la percepción de los cuidadores comían "demasiado" $(\beta=0,68)$, comían en un "tiempo adecuado" $(\beta=0,86)$ o "muy rápido" $(\beta=0,65)$.

Por el contrario, se encontró disminución significativa

\section{TABLA 2}

Antecedentes patológicos de las madres de una muestra de niños y niñas de los Hogares Infantiles del ICBF asociados al puntaje z IMC para edad y sexo. Floridablanca, Colombia, 2012.

\begin{tabular}{|c|c|c|c|c|c|c|}
\hline Variable & $\mathrm{n}$ & $\%$ & IC95\% & Coeficiente Beta & IC95\% & Valor $p$ \\
\hline \multicolumn{7}{|l|}{ Obesidad } \\
\hline No & 119 & 67,6 & $60,6-74,6$ & & & \\
\hline $\mathrm{Si}$ & 32 & 18,2 & $12,4-23,9$ & 0,33 & 0,$08 ; 0,58$ & 0,010 \\
\hline Sin información/no sabe & 25 & 14,2 & $9,0-19,4$ & 0,26 & $-0,02 ; 0,53$ & 0,067 \\
\hline \multicolumn{7}{|l|}{ Diabetes } \\
\hline No & 100 & 56,8 & $49,4-64,2$ & & & \\
\hline $\mathrm{Si}$ & 51 & 29,0 & $22,2-35,7$ & $-0,49$ & $-0,70 ;-0,27$ & 0,000 \\
\hline Sin información/no sabe & 25 & 14,2 & $9,0-19,4$ & 0,04 & $-0,24 ; 0,32$ & 0,758 \\
\hline \multicolumn{7}{|l|}{ Enfermedades cardiovasculares } \\
\hline No & 95 & 54,0 & $46,5-61,4$ & & & \\
\hline $\mathrm{Si}$ & 50 & 28,4 & $21,7-35,1$ & 0,02 & $-0,20 ; 0,24$ & 0,850 \\
\hline Sin información/no sabe & 31 & 17,6 & $11,9-23,3$ & 0,42 & 0,$16 ; 0,68$ & 0,002 \\
\hline \multicolumn{7}{|l|}{ Hipertrigliceridemia } \\
\hline No & 110 & 62,5 & $55,3-69,7$ & & & \\
\hline $\mathrm{Si}$ & 36 & 20,5 & $14,4-26,5$ & $-0,09$ & $-0,33 ; 0,15$ & 0,476 \\
\hline Sin información/no sabe & 30 & 17,0 & $11,4-22,7$ & 0,07 & $-0,19 ; 0,33$ & 0,602 \\
\hline \multicolumn{7}{|l|}{ Hipercolesterolemia } \\
\hline No & 104 & 59,1 & $51,8-66,4$ & & & \\
\hline $\mathrm{Si}$ & 43 & 24,4 & $18,0-30,8$ & $-0,10$ & $-0,34 ; 0,12$ & 0,349 \\
\hline Sin información/no sabe & 29 & 16,5 & $10,9-22,0$ & 0,03 & $-0,24 ; 0,30$ & 0,817 \\
\hline \multicolumn{7}{|l|}{ Hipertensión arterial } \\
\hline No & 91 & 51,7 & $44,2-59,2$ & & & \\
\hline $\mathrm{Si}$ & 58 & 33,0 & $25,9-40,0$ & $-0,32$ & $-0,53 ;-0,11$ & 0,003 \\
\hline Sin información/no sabe & 27 & 15,3 & $10,0-20,7$ & $-0,05$ & $-0,33 ; 0,22$ & 0,694 \\
\hline \multicolumn{7}{|c|}{ Clasificación del IMC de la madre } \\
\hline Normal & 69 & 39,2 & 31,$9 ; 46,4$ & & & \\
\hline Exceso & 85 & 48,3 & 40,$8 ; 55,8$ & 0,50 & 0,$31 ; 0,71$ & 0,000 \\
\hline
\end{tabular}


TABLA 3

Estilos de crianza relacionados con alimentación de una muestra de niños y niñas de los Hogares Infantiles del ICBF asociados al puntaje z IMC para edad y sexo. Floridablanca, Colombia, 2012.

$$
\text { Variable }
$$

$\mathrm{n}$

$\%$

IC95\%

Coeficiente Beta

IC95\%

Valor $\mathrm{p}$

En las comidas principales, ofrece cantidades

adicionales a la brindada inicialmente

No

$\mathrm{Si}$

Acciones mientras el niño(a) está comiendo

Se dedica a comer con el niño(a)

Hace otra actividad

Acciones cuando el niño(a) deja de comer y el

cuidador cree que no ha comido lo suficiente

No le dice nada/Siempre come

Lo motiva/Ofrece otro alimento/

Guarda/Conversa

Lo presiona/Chantaje/Regaño

Acciones cuándo el niño no come lo que se le ofrece

Siempre Come/No le hace nada

Lo motiva/Ofrece otro alimento/

Guarda/Conversa.

Lo presiona/Chantaje/Regaño

Formas para promover el consumo de alimentos

Palabras-dialogo

Motivación/Juegos-Regalos

Presión/Chantaje/Regaño

No lo motiva/No es necesario

Acompaña al niño (a) mientras consume los alimentos

Madre o padre

Familiar

Solo/otra persona no familiar

Prepara alimento

Madre

Abuela

Otro

Ofrece la alimentación lunes a viernes

Madre

Abuela

Otro

Ofrece la alimentación sábado

Madre

Abuela

Otro

Ofrece la alimentación domingo

Madre

Abuela

Otro

Decide el tipo de alimentos que el niño(a) consume

Madre

Abuela

Otro

Conducta habitual a la hora de comer

Come con entusiasmo

Rechaza la comida/come sin apetito/

Juega con la comida

Percepción del tiempo que el niño(a) emplea en

consumir los alimentos

Come despacio

Come en un tiempo adecuado

Come rápido

Percepción del apetito del niño(a) cuando está sano

Come suficiente

Come demasiado

Come poco

\begin{tabular}{|c|c|c|c|c|c|}
\hline 107 & 60,8 & $53,5-68,1$ & & & \\
\hline 68 & 38,6 & $31,4-45,9$ & $-0,27$ & $-0,47 ;-0,08$ & 0,005 \\
\hline 166 & 94,3 & $90,9-97,7$ & & & \\
\hline 8 & 4,5 & $1,4-7,7$ & $-0,19$ & $-0,65 ; 0,29$ & 0,215 \\
\hline 26 & 14,8 & $9,4-20,0$ & & & \\
\hline 112 & 63,6 & $56,5-70,8$ & $-1,15$ & $-1,41 ;-0,88$ & 0,000 \\
\hline 33 & 18,8 & $12,9-24,6$ & $-1,03$ & $-1,35 ;-0,72$ & 0,000 \\
\hline 113 & 64,2 & $57,1-71,4$ & & & \\
\hline 21 & 11,9 & $7,0-16,8$ & $-0,05$ & $-0,26 ; 0,35$ & 0,759 \\
\hline 40 & 22,7 & $16,5-29,0$ & $-0,01$ & $-0,25 ; 0,22$ & 0,904 \\
\hline 73 & 41,5 & $34,1-48,8$ & & & \\
\hline 31 & 17,6 & $11,9-23,3$ & $-0,18$ & $-0,45 ; 0,09$ & 0,201 \\
\hline 57 & 32,4 & $25,4-39,4$ & 0,0003 & $-0,22 ; 0,22$ & 0,998 \\
\hline 12 & 6,8 & $3,1-10,6$ & 0,50 & 0,$11 ; 0,90$ & 0,013 \\
\hline 74 & 42,1 & $34,7-49,4$ & & & \\
\hline 79 & 45,0 & $37,5-52,3$ & 0,76 & 0,$57 ; 0,96$ & 0,000 \\
\hline 22 & 12,5 & $7,6-17,4$ & 0,81 & 0,$51 ; 1,10$ & 0,000 \\
\hline 105 & 59,7 & $52,3-67,0$ & & & \\
\hline 49 & 27,8 & $21,2-34,5$ & 0,25 & $-0,03 ; 0,47$ & 0,025 \\
\hline 21 & 11,9 & $7,1-16,8$ & $-0,11$ & $-0,41 ; 0,19$ & 0,474 \\
\hline 106 & 60,2 & $52,9-67,5$ & & & \\
\hline 46 & 26,1 & $19,6-32,7$ & 0,17 & $-0,06 ; 0,39$ & 0,148 \\
\hline 23 & 13,1 & $8,0-18,1$ & $-0,08$ & $-0,38 ; 0,21$ & 0,575 \\
\hline 107 & 60,8 & $53,5-68,1$ & & & \\
\hline 43 & 24,4 & $18,0-30,8$ & 0,26 & 0,$04 ; 0,49$ & 0,022 \\
\hline 25 & 14,2 & $9,0-19,4$ & 0,40 & 0,$12 ; 0,68$ & 0,005 \\
\hline 126 & 71,6 & $64,9-78,3$ & & & \\
\hline 29 & 16,5 & $10,9-22,0$ & 0,31 & 0,$05 ; 0,57$ & 0,019 \\
\hline 19 & 10,8 & $6,2-15,4$ & 0,37 & 0,$06 ; 0,68$ & 0,020 \\
\hline 125 & 71,0 & $64,3-77,8$ & & & \\
\hline 31 & 17,6 & $11,9-23,3$ & 0,11 & $-0,14 ; 0,36$ & 0,385 \\
\hline 19 & 10,8 & $6,2-15,4$ & 0,48 & 0,$17 ; 0,80$ & 0,002 \\
\hline 112 & 63,6 & $56,5-70,8$ & & & \\
\hline 63 & 35,8 & $28,6-42,9$ & $-0,36$ & $-0,56 ;-0,16$ & 0,000 \\
\hline 76 & 43,2 & $35,7-50,6$ & & & \\
\hline 88 & 50,0 & $42,5-57,5$ & 0,86 & 0,$68 ; 1,06$ & 0,000 \\
\hline 11 & 6,3 & $2,6-98,6$ & 0,65 & 0,$26 ; 1,04$ & 0,001 \\
\hline 133 & 75,6 & $69,2-81,9$ & & & \\
\hline 20 & 11,4 & $6,6-16,1$ & 0,68 & 0,$39 ; 0,98$ & 0,000 \\
\hline 22 & 12,5 & $7,6-17,4$ & $-0,68$ & $-0,96 ;-0,39$ & 0,000 \\
\hline
\end{tabular}


del puntaje z del IMC para la edad y sexo en los niños que en las comidas principales se les ofrecía comida adicional a la brindada inicialmente $(\beta=-0,27)$, a los niños que cuando dejaban de comer y que el cuidador creía que no había comido lo suficiente, lo motivaba $(\beta=-1,15)$ o lo presionaba $(\beta=-1,03)$; en los niños que rechazaban la comida/comían sin apetito o jugaban con la comida $(\beta=-0,36)$ y en aquellos niños que según la percepción de los cuidadores "comían poco" ( $\beta=-$ 0,68 ) (tabla 3).

En el análisis multivariado se encontró un aumento significativo del puntaje $z$ para la edad y sexo con la edad $(\beta=0,02)$ y sexo masculino $(\beta=0,27)$. Así mismo, cuando la madre se dedicaba a oficios del hogar comparado con las que trabajaban, el promedio del puntaje $z$ del IMC para edad y sexo aumentó 0,29 . Si la madre tenía exceso de peso el promedio del puntaje z del IMC de los preescolares aumentaba 0,20 comparado con el promedio del grupo de madres con peso normal. Los preescolares acompañados por los familiares aumentaron el promedio del puntaje $z$ en 0,49 y niños que consumían los alimentos solos o con otra persona no familiar aumentaban el puntaje promedio en 0,40 comparado con el promedio de los preescolares acompañados por madre o padre. En los preescolares a quienes les preparaba los alimentos la abuela aumentó el promedio del puntaje z en 0,55 comparado con

TABLA 4

Factores asociados al puntaje z del IMC para edad y sexo. Análisis Multivariado usando Modelo lineal Generalizado multivariado. $n=148$.

\begin{tabular}{|c|c|c|c|c|}
\hline Variable & Coeficiente Beta & IC95\% & Valor $\mathrm{p}$ & Variable \\
\hline Edad (meses) & 0.02 & 0.01 & 0.03 & 0.000 \\
\hline \multicolumn{5}{|l|}{ Sexo $n(\%)$} \\
\hline \multicolumn{5}{|l|}{ Femenino } \\
\hline Masculino & 0.27 & 0.08 & 0.47 & 0.006 \\
\hline \multicolumn{5}{|l|}{ Ocupación de la madre } \\
\hline \multicolumn{5}{|l|}{ Trabajando } \\
\hline Buscando Trabajo/Estudiando & -0.43 & -0.81 & -0.05 & 0.028 \\
\hline Oficios del hogar & 0.29 & 0.00 & 0.58 & 0.053 \\
\hline \multicolumn{5}{|l|}{ Clasificación del IMC de la madre } \\
\hline \multicolumn{5}{|l|}{ Normal } \\
\hline Exceso de peso & 0.20 & 0.01 & 0.39 & 0.043 \\
\hline \multicolumn{5}{|l|}{ Antecedente de hipertensión arterial de la madre } \\
\hline \multicolumn{5}{|l|}{ No } \\
\hline $\mathrm{Si}$ & -0.26 & -0.47 & -0.05 & 0.015 \\
\hline No sabe/Sin información- debía analizarse estos? & & & & \\
\hline O se elimina de aca? & -0.02 & -0.30 & 0.27 & 0.898 \\
\hline \multicolumn{5}{|l|}{$\begin{array}{l}\text { Acciones cuando el niño(a) deja de comer y el cuidador } \\
\text { cree que no ha comido lo suficiente }\end{array}$} \\
\hline \multicolumn{5}{|l|}{ No le dice nada/Siempre come } \\
\hline Lo motiva/ofrece otro alimento/guarda/conversa & -0.59 & -0.90 & -0.27 & 0.000 \\
\hline Lo presiona/chantaje/regaño & -0.55 & -0.91 & -0.19 & 0.003 \\
\hline \multicolumn{5}{|l|}{ Acompaña al niño (a) mientras consume los alimentos } \\
\hline \multicolumn{5}{|l|}{ Madre o padre } \\
\hline Familiar & 0.49 & 0.28 & 0.69 & 0.000 \\
\hline Solo/otra persona no familiar & 0.40 & 0.03 & 0.78 & 0.034 \\
\hline \multicolumn{5}{|l|}{ Preparar alimento } \\
\hline \multicolumn{5}{|l|}{ Madre } \\
\hline Abuela & 0.55 & 0.25 & 0.85 & 0.000 \\
\hline Otro & -0.09 & -0.39 & 0.21 & 0.548 \\
\hline \multicolumn{5}{|l|}{ Decide el tipo de alimentos que el niño(a) consume } \\
\hline Madre & & & & \\
\hline Abuela & -0.35 & -0.71 & 0.00 & 0.052 \\
\hline Otro & 0.47 & 0.12 & 0.83 & 0.009 \\
\hline \multicolumn{5}{|l|}{$\begin{array}{l}\text { Percepción del tiempo que el niño(a) emplea en } \\
\text { consumir los alimentos }\end{array}$} \\
\hline \multicolumn{5}{|l|}{ Come despacio } \\
\hline Come en un tiempo adecuado & 0.54 & 0.33 & 0.75 & 0.000 \\
\hline Come rápido & 0.17 & -0.26 & 0.59 & 0.439 \\
\hline \multicolumn{5}{|l|}{$\begin{array}{l}\text { Percepción de la cantidad de alimento consumida } \\
\text { cuando está sano }\end{array}$} \\
\hline \multicolumn{5}{|l|}{ Come suficiente } \\
\hline Come demasiado & 0.51 & 0.22 & 0.81 & 0.001 \\
\hline Come poco & -0.34 & -0.61 & -0.06 & 0.017 \\
\hline Constante & -0.92 & -1.61 & -0.23 & 0.009 \\
\hline
\end{tabular}


la preparación de alimentos realizada por la madre. En quienes según la percepción del cuidador "comen en un tiempo adecuado" el promedio del puntaje z aumentó 0,54 comparado con "comer despacio". En los preescolares que "comen demasiado", según la percepción del cuidador, el promedio del puntaje z aumentó 0,51 comparado con el promedio de los preescolares que "come suficiente". Cada coeficiente Beta se ajustó por las demás variables (tabla 4).

Por el contrario, el promedio del puntaje z disminuyó en los preescolares en las siguientes situaciones, cuando las madres estaban buscando trabajo o estudiando disminuyó 0,43 comparado con las madres que estaban trabajando, cuando la madre presentaba antecedentes de hipertensión arterial el puntaje z disminuyó en 0,26 comparado con los preescolares cuya madre no presentó antecedentes de hipertensión arterial; también disminuyó cuando los preescolares dejaban de comer y el cuidador considerando que no ha comido lo suficiente los motivaba o los presionaba, en el primer grupo disminuyó en 0,59 y en el segundo en 0,55, comparado con el grupo al que no le decían nada o que siempre comía (tabla 4).

\section{DISCUSIÓN}

La asociación con el aumento significativo del IMC, estuvo dada por la edad del preescolar. Benton (21), afirma que hay doble riesgo de obesidad a los 35 años en quienes fueron obesos a la edad de 1 a 6 años y un riesgo de 5 a 10 veces en quienes lo fueron a la edad de 10 - 14 años, contrario a lo observado por Heitzinger quien plantea la necesidad de investigar más sobre la relación edad y exceso de peso (22). También se encontró aumento significativo del puntaje z del IMC en los varones, resultados similares se observaron en la ENSIN 2005 y 2010 (4), y los encontrados en población escolar de México (23), Argentina (24) y Brasil (25).

El exceso de peso en las madres fue otro factor con asociación positiva a un IMC mayor en los niños, así como lo reportado por Loaiza et al. (26), Birch et al. (27) y Puente et al. (28), posiblemente debido a la carga genética o a la transmisión de modelos y patrones de alimentación poco saludables, o una mezcla de estos factores (28).

El acompañamiento a la hora de las comidas fue una variable del ambiente alimentario que se exploró en este estudio, se encontró un mayor IMC en los preescolares que se alimentaron solos o que estuvieron acompañados por personas diferentes a sus padres. Otras investigaciones con este grupo de edad, han reportado como el poco control ejercido por los padres en el momento de las comidas se asoció a obesidad $(29,30)$, resultados similares se observaron en adolescentes (31).

La responsabilidad de la preparación de los alimentos y la asociación con estado nutricional es un factor poco estudiado. Este estudio encontró que en una cuarta parte de la población, la abuela es la responsable de preparar y ofrecer la alimentación al preescolar y que el IMC fue mayor cuando ella lo realizaba, comparado cuando la madre era la responsable. Resultados similares se encontraron en otros estudios $(32,33)$, donde las puntuaciones de IMC de los niños, fueron inferiores cuando la comida principal era elaborada por las madres. Es probable que la situación se deba a que la porción ofrecida por la abuela sea mayor a la ofrecida por la madre. En este estudio se exploró de manera cualitativa, la cantidad consumida teniendo en cuenta la percepción del cuidador y se encontró que los niños que "comen demasiado" comparado con los que "comen suficiente" tienen mayor IMC y los que "comen poco" tienden a un menor IMC. Es importante realizar estudios posteriores que permitan determinar si existe diferencia entre el tamaño de la porción ofrecida al preescolar, según el tipo de cuidador.

Se ha evidenciado la asociación del tamaño de la porción consumida con el peso corporal (27), y la importancia que tiene como medio eficaz y ágil para realizar la educación nutricional, es así como muchos países han implementado dentro de las Guías alimentarias, la imagen del plato con porciones de alimentos como el ícono para impactar el consumo de saludable (34).

Respecto a los factores asociados con la disminución del $I M C$, se encontró que es la influencia ejercida por el cuidador en el momento de las comidas principales, sin importar el tipo de influencia la que se asocia a un menor IMC. Este factor es muy controvertido, Clark HR et al., en una revisión sistemática realizada sobre las pautas de crianza relacionadas con la alimentación, encontró que la evidencia es inconsistente, la regulación externa del consumo de alimentos puede ser un mecanismo contraproducente o favorecedor de la cantidad consumida y del peso corporal $(8,33)$.

La limitación de este estudio consiste en la tasa de no respuesta de algunas variables, por lo cual el análisis multivariado se redujo a una muestra de 146 niños, esto puede explicarse porque algunas preguntas hacían referencia a aspectos particulares de los padres, además no fue posible obtener las mediciones antropométricas en la totalidad de ellos, uno de los factores a considerar es que 38,6\% de los niños no vivía con ambos padres.

Se concluye que el aumento del IMC en los preescolares estudiados se asocia positivamente con factores del ambiente alimentario como, persona responsable de la preparación de alimentos, acompañamiento durante las comidas principales, tiempo empleado en alimentarse y la cantidad consumida. Otras variables asociadas fueron, edad del preescolar, sexo masculino, ocupación de las madres (dedicadas al hogar) y exceso de peso de las madres. De otra parte se encontró que la disminución del IMC se asoció con la influencia ejercida por los padres o cuidadores para que los niños consuman los alimentos.

Se ha evidenciado la influencia que tiene la educación nutricional de los padres en los hábitos saludables de sus familias $(10,33)$, los resultados de estudio permitirán orientar los proyectos educativos desarrollados en la primera infancia, teniendo en cuenta las condiciones ambientales y las pautas de crianza.

La relevancia de este trabajo radica en que existen pocos trabajos en el ámbito internacional que hayan abordado los factores ambientales y pautas de crianza asociados al IMC en preescolares, además no se conocen estudios a nivel nacional.

\section{RESUMEN}

El objetivo del estudio fue determinar la asociación de factores ambientales y estilos de crianza con el puntaje $z$ del Índice de Masa Corporal (IMCZ) en preescolares de los Hogares Infantiles de Floridablanca, Colombia. Una muestra aleatoria de 176 preescolares de 3 a 6 años fueron medidos y pesados; sus padres o cuidadores fueron medidos y respondieron una encuesta. El modelo lineal generalizado mostró que el IMCZ aumenta significativamente con la edad $(\beta=0,02)$ y sexo del preescolar (masculino $\beta=0,27$ ), ocupación (ama de casa $\beta=0,29)$ y sobrepeso de la madre $(\beta=0,20)$, persona que lo acompaña mientras come (familiares $\beta=0,49$; ninguno o no familiares $\beta=0,40$ ), persona que prepara los alimentos (abuela $\beta=0,55)$, comer en un tiempo adecuado $(\beta=0,54)$, y comer demasiado $(0,51)$. El IMCZ disminuye significativamente con la ocupación de la madre (buscando trabajo o 
estudiante $\beta=-0,43)$, antecedentes de hipertensión arterial maternos $(\beta=-0,26)$, motivación para comer $(\beta=-0,59), y$ presión para comer $(\beta=-0,55)$.

Palabras clave: Pautas de crianza, factores de riesgo, indice de masa corporal, obesidad pediátrica, conducta alimentaria.

\section{BIBLIOGRAFÍA}

1. Ebbeling CB, Pawlak DB, Ludwig DS. Childhood obesity: public-health crisis, common sense cure. Lancet. 2002;360(9331):473-82.

2. Han J, Lawlor D, Kimm S. Childhood obesity Review Article. Lancet. 2010; 375(9727):1737-48.

3. Rivera JA, González T, Pedraza LS, Aburto TC, Sánchez TG, Martorell R. Childhood and adolescent overweight and obesity in Latin America: a systematic review. Lancet Diabetes Endocrinol. 2014;2(4):321-32.

4. Instituto Colombiano de Bienestar Familiar (2010). Encuesta Nacional de la Situación Nutricional en Colombia-ENSIN 2010-. [Citado: 29 de abril de 2014] Disponible en: http:// www.icbf.gov.co/portal/page/portal/Portal/CBF/NormatividadC/ENSIN1/ENSIN2010/LibroENSIN2010.pdf

5. Azula L, Pérez E, Sandoval M, Schneider S. Epidemiologia del sobrepeso y la obesidad en niños y adolescentes. Rev Vía Cáted Med. 2008; 179:16-20.

6. Perry C, Luepker R, Murray D. Kurth C. Parent involvement with Children's Health Promotions: The Minnesota Home Team. Am J Public Health. 1998;78:1156-60.

7. Moraga F, Rebollo MJ, Bórquez P, Cáceres J, Castillo C. Tratamiento de la obesidad infantil: Factores pronósticos asociados a una respuesta favorable. Rev Chil Pediatr. 2003; 74 (4):374-80.

8. Peungposop $N$, Junprasert $T$. A research review on type of child rearing practices and childhood obesity. Inter J Behear Sci. 2014; 9 (1): 45-54.

9. Blissett J, Bennett C. Cultural differences in parental feeding practices and children's eating behaviours and their relationships with child BMI: a comparison of Black AfroCaribbean, White British and White German samples. Eur J Clin Nutr. 2013;67(2):180-4.

10. Sánchez J, Ruiter I, Jiménez J. Individual, family and environmental factors associated with pediatric excess weight in Spain: a cross-sectional study. BMC Pediatrics. 2014;14:3. Disponible en: www.biomedcentral.com/1471-2431/14/3.

11. Subcomisión de Epidemiología y Comité de Nutrición. Consenso sobre factores de riesgo de enfermedad cardiovascular en pediatría. Obesidad. Arch Argent Pediatr. 2005; 103(3):262-81.

12. Reilly J, Armstrong J, Dorosty A, Emmett P, Ness A, Rogers $A$, et al. Early life risk factors for obesity in childhood: cohort study. BMJ. 2005; 330:1357-63.

13. Informes técnicos del estado nutricional de los usuarios de los Hogares Infantiles del ICBF. Floridablanca, Santander, Colombia. 2012.

14. Rosero LM. Estratificación socioeconómica como instrumento de focalización. Rev Econ Des. 2004; 3(1): 53-67.

15. Organización Mundial de la Salud (2010). Software ANTHRO, v 3.1.Ginebra, Suiza.

16. Lauritsen J. (Ed.) EpiData Data Entry, Data Management and basic Statistical Analysis System. Odense Denmark, EpiData Association, 2000-2008. Disponible en: www. epidata.dk.
17. StataCorp. Stata Statistical Software: Release 12. College Station, TX: StataCorp LP. 2011.

18. Norman G, Streiner D. Bioestadística. Madrid: Mosby/ Doyma Libros, 1996.

19. Fonseca M, Andreozzi V, Faerstein E, Chor D, Carvalho M. Alternatives in modeling of body mass index as a continuous response variable and relevance of residual analysis. Cad. Saúde Pública. 2008; 24(2):473-8.

20. Ministerio de Salud de la República de Colombia. Resolución 008430 de 1993. Bogotá; 4 de octubre de 1993.

21. Benton D. Role of parents in the determination of the food preferencies of children and the development of obesity. Int J Obes Relat Metab Disord. 2004;28(7):858-69.

22. Heitzinger $K$, Vélez J, Parra S, Barbosa C, Fitzpatrick A. Caregiver perceptions of child nutritional status in Magallanes, Chile. Obe Res Clin Pract 2005; 8 (1): e98-e105.

23. Instituto Nacional de Salud Pública. Encuesta Nacional de Salud y Nutrición 2006 (ENSANUT). Cuernavaca, Morelos, México, 2008.

24. Kovalskys I, Bay L, Herscovici C, Berner E. Prevalencia de obesidad en una población de 10 a 19 años en la consulta pediátrica. Rev Chil Pediatr. 2005;76:324-5.

25. Ogden C, Carrol M, Kit B, Flegal K. Prevalence de obesity and trends in body mass index among US children and adolescents, 199-2010. JAMA. 2012; 307(5):483-90.

26. Loaiza M, Atalah E. Factores de riesgo de obesidad en escolares de primer año básico de Punta Arenas. Rev Chil Pediatr. 2006; 77(1):20-6.

27. Birch LL, Fisher JO. Development of Eating Behaviors Among Children and Adolescents. Pediatrics. 1998;101(3):539-49.

28. Puente $M$, Ricardo $T$, Fernández $R$. Factores de riesgo relacionados con la obesidad en niñas y niños menores de 5 años. Rev Cubana Alim Nutr. 2013;17(7):1065-71.

29. Chacón A. Control y presión al comer en madres de preescolares costarricenses, y su relación con la clase social, la escolaridad, las prácticas de alimentación temprana y el índice de masa corporal de sus hijos e hijas. Pobl Salud Mesoam. 2011;9(1): Disponible en http://ccp.ucr.ac.cr/ revista/volumenes/9/9-1/9-1-1/9-1-1.pdf.

30. Hoerr SL, Hughes SO, Fisher JO, Nicklas TA, Liu Y, Shewchuk RM. Associations among parental feeding styles and children's food intake in families with limited incomes. Int J Behav Nutr Phys Act. 2009; 6: 55. Disponible en: http:// www.ncbi.n/m.nih.gov/pmc/articles/PMC2739505/ $p d f / 1479-5868-6-55 . p d f$

31. Osorio-Murillo O, Amaya-Rey M. La alimentación de los adolescentes: el lugar y la compañía determinan las prácticas alimentarias. Aquichán. 2011; 11(2): 199-216.

32. González E, Aguilar M, García C, García P, Alvarez J, Padilla $C$ et al. Influencia del entorno familiar en el desarrollo del sobrepeso y la obesidad en una población de escolares de Granada (España). Nutr. Hosp. 2012; 27 (1):177-84.

33. Clark H, Goyder E, Bissell P, Blank L, Peters. J. How do parents' child-feeding behaviours influence child weight? Implications for hildhood obesity policy. J Public Health (Oxf). 2007;29(2):132-41.

34. Katamay SW, Esslinger KA, Vigneault $M$, JohnstonJL, Junkins BA, Robbins LG, et. al. Eating Well with Canada's Food Guide. Develop Food Intake Pattern Nutr Rev. 2007;65(4):155-66. 